\title{
Wideband receiver design using frequency-dependent magnitude/phase mismatch
}

\author{
Chi-Hao Chenga) \\ Department of Electrical and Computer Engineering, Miami University, \\ Oxford, Ohio, USA \\ a) chengc@miamioh.edu
}

Abstract: We propose a digital wideband receiver scheme covering multiple Nyquist zones by exploiting phase and magnitude mismatch in the conversion from real-valued signals to complex-valued signals. The digital wideband receiver is designed to detect uncooperative signals. The magnitude/phase mismatch due to an imperfect hybrid coupler is often considered undesirable and needs to be compensated. However, by exploiting the frequency-dependent magnitude/phase mismatch phenomenon, we can distinguish signals from different Nyquist zones. Simulation results demonstrate that, using carefully designed magnitude/phase mismatch, we can detect signals over multiple Nyquist zones. The proposed method can lead to a new wideband receiver design.

Keywords: sub-Nyquist sampling, I/Q imbalance, wideband receiver

Classification: Electronic instrumentation and control

\section{References}

[1] J. Tsui: Digital Techniques for Wideband Receivers (Artech House, 2001) 2nd ed.

[2] R. Wiley: ELINT the Interception and Analysis of Radar Signals (Artech House, 2006).

[3] J. Tsui: Special Design Topics in Digital Wideband Receivers (Artech House, 2010).

[4] S. K. Mitra: Digital Signal Processing A Computer-Based Approach (McGraw-Hill, 2006) 3rd ed.

[5] L. Po, Z. Yu and C. Z. Ping: Proc. IET Radar Conference (2009). DOI:10. 1049/cp.2009.0302

[6] K.-H. Lin, H.-L. Lin, S.-M. Wang and R. C. Chang: Proc. IEEE ISCAS (2006) 3534. DOI:10.1109/ISCAS.2006.1693389

[7] J.-Y. Yu, M.-F. Sun, T.-Y. Hsu and C.-Y. Lee: Proc. IEEE ISCAS (2005) 6030. DOI:10.1109/ISCAS.2005.1466014

[8] K.-P. Pun, J. Franca and C. Azeredo-Leme: Proc. IEEE ISCAS (2000) 661. DOI:10.1109/ISCAS.2000.857556

[9] C.-H. Cheng, L. L. Liou, D. M. Lin, J. B. Tsui and H.-M. Tai: accepted by IET Radar, Sonar, and Navigation (2014) http://digital-library.theiet.org/ content/journals/10.1049/iet-rsn.2013.0123.

[10] C.-H. Cheng, D. M. Lin, L. L. Liou and J. B. Tsui: IEEE Trans. Aerosp. Electron. Syst. 48 (2012) 3318. DOI:10.1109/TAES.2012.6324709 


\section{Introduction}

The purpose of a digital wideband receiver is to detect unknown number of un-cooperative signals over a broad bandwidth $[1,2,3]$. Unlike a communication receiver which has information about signal's frequency, modulation type, and etc., this information is usually unavailable to the digital wideband receiver and the digital wideband receiver needs to extract signal characteristics such as frequency, power, and etc. in real-time. Nyquist theory states that, sampling an analog signal at sampling frequency, $F_{s}$, the signal can be determined without ambiguity if its frequency is less than half of sampling rate $\left(F_{s} / \mathscr{2}\right.$, also known as the Nyquist rate), i.e. in the first Nyquist zone $\left(0, F_{s} / 2\right)$ [4]. As the result, it is a common practice to divide the working bandwidth of a digital wideband receiver into several bands each of which has a processing unit as shown in Fig. 1. In this receiver, the input signal is down-converted to the baseband by different down-converters working in different frequencies. In each band, an ADC is used to digitize signals, a Blackman window is used to reduce spectral leakage [1], and Fast Fourier Transform (FFT) is conducted to get signal's spectral information. An analysis device then extracts signal's characteristics based on outputs of FFT modules. To cover the same bandwidth, the slower the A/D converter's sampling rate, the more ADC and processing units are necessary. Besides, due to the aliasing effects, the signal whose frequency is around Nyquist rates can cause false alarm, i.e. the detection of non-existent signals [1]. Therefore, the working bandwidth of each band is less than Fs/2.

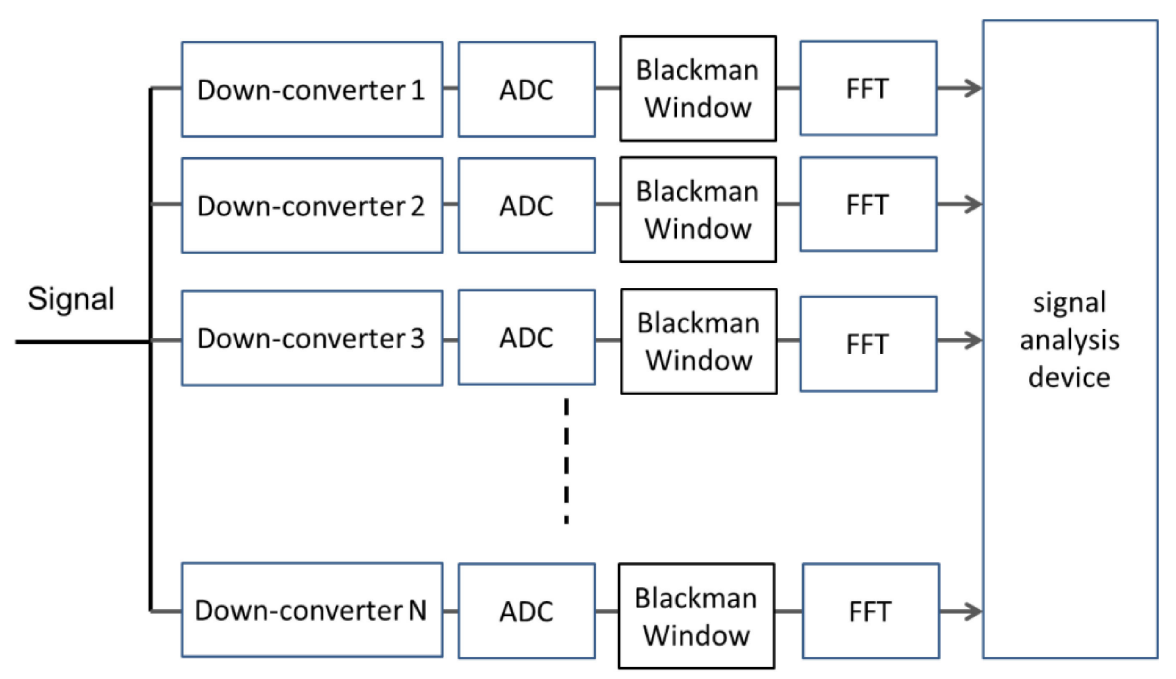

Fig. 1. Conventional digital wideband receiver diagram for a wide bandwidth coverage. A similar diagram can be found in Ref. [5].

It is known that, by converting a real-valued signal to a complex-valued signal with a 90 degree coupler, an asymmetrical spectrum is generated and the receiver's working bandwidth can be doubled and aliasing effects mentioned previously can be reduced [1]. However, the image signal generated during the imperfect conversion process due to the coupler's magnitude and 
phase imbalance can cause a false alarm. Therefore, the application of complex-valued digital wideband receiver is limited. Although the magnitude and phase imbalance is a common issue in communication systems, due to the facts that wideband receiver does not assume signal frequency and signals to be detected might have very short duration, the imbalance compensation methods developed for communication receiver are not suitable for wideband receiver $[6,7,8]$. Recently, the author co-developed an algorithm to compensate frequency dependent magnitude/phase mismatch for wideband receiver application [9]. With a magnitude/phase imbalance compensator, the working bandwidth of a wideband receiver can be doubled. In this article, we would like to further expand the receiver bandwidth beyond the second Nyquist zone. By intentionally introducing frequency-dependent magnitude/phase mismatch and using the mismatch compensation, the signal's Nyquist zone can be identified. Thus, it is possible to expand the receiver's working bandwidth beyond the second Nyquist zone. In other words, the undesired magnitude/phase mismatch can be intentionally used to increase a receiver's working bandwidth. To the best of the author's knowledge, such a method has never been proposed and can lead to a new design of digital wideband receiver.

\section{Signal conversion}

An I/Q channel receiver is illustrated in Fig. 2(a). A real-valued signal passes through a 90 degree hybrid coupler which generates two components: in-phase (I) and quadrature (Q). A perfect hybrid coupler introduces an exact 90 degree phase shift $(\phi=0$ in Fig. 2(a)) and its in-phase and quadrature components have the same magnitude $(\mathrm{k}=1$ in Fig. 2(a)). A realistic coupler does not generate an exact 90 degree phase shift $(\phi \neq 0$ in Fig. 2(a)) and its inphase and quadrature components have different magnitudes $(k \neq 1$ in Fig. 2(a)). This phenomenon is referred to as magnitude/phase mismatch. Using a cosine signal of $2.003 \mathrm{GHz}$ frequency sampled at $2.56 \mathrm{GHz}$ as the input signal, we conducted a 256-point FFT upon the original real-valued signal and the coupler output. The coupler output is treated as a complex-valued signal (real part: in-phase, imaginary part: quadrature). The spectra of real-valued signal, complex-valued signal generated by a perfect coupler, and complexvalued signal generated by an imperfect coupler are illustrated in Fig. 2(b). As shown in Fig. 2(b), the peak at the first Nyquist zone is removed by a perfect coupler, so signals in the first and second Nyquist zones can be distinguished and receiver's working bandwidth can thus be doubled. However, when an imperfect coupler is used $\left(\phi=4.0224^{\circ}\right.$ and $\mathrm{k}=0.9196$ in this case), a "ghost" image re-appears in the first Nyquist zone and we cannot distinguish an image signal caused by an imperfect coupler from a valid but weaker signal. It becomes a serious concern because the purpose of the digital wideband receiver is to detect unknown numbers of signals with unknown frequency and power $[1,2,3]$.

The magnitude/phase mismatch is not a new problem. It has been wellstudied in communication systems $[6,7,8]$. However, mismatch compensation 


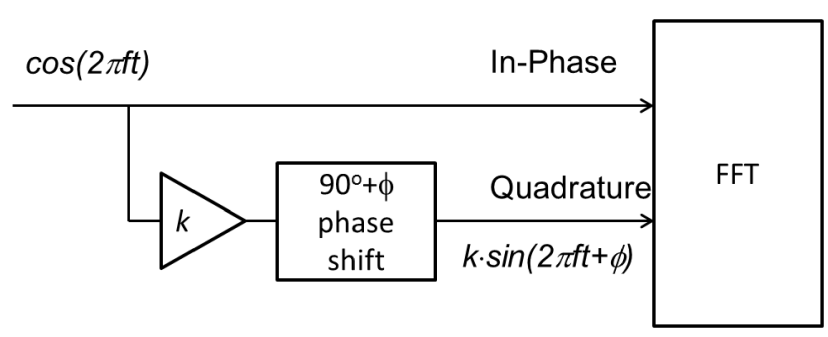

(a)

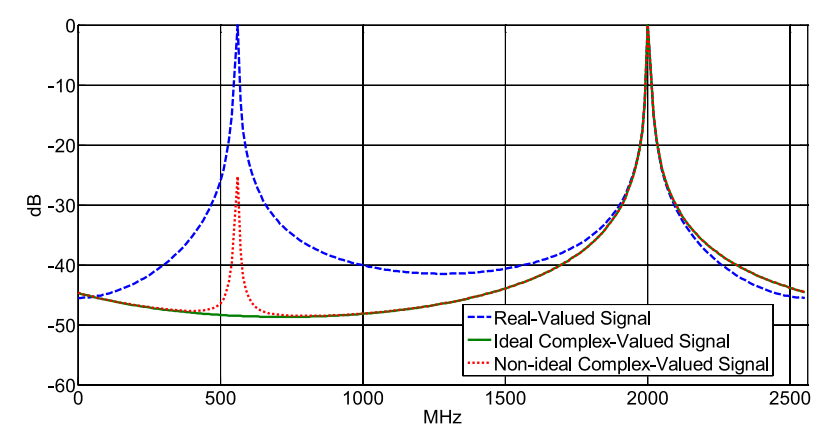

(b)

Fig. 2. (a) I/Q channel based wideband receiver diagram (b) Spectra of real-valued signal, complex-valued signal generated by a perfect coupler, complex signal generated by an imperfect coupler. (Sampling rate: $2.56 \mathrm{GHz})$

schemes developed by communication engineers either assume the magnitude/phase mismatch is a constant value $[6,7]$ or signals of interests are confined in the same Nyquist zone [8]. Recently, the author co-developed a new magnitude/phase mismatch compensation scheme designed to compensate frequency-dependent magnitude/phase mismatch over two Nyquist zones [9]. The input and output relation of the equalizer is given in Eq. (1)

$$
Z_{\text {out }, k}=\sum_{m=-M}^{M} c_{m} z_{i n, k-m}+\sum_{m=-M}^{M} d_{m} z_{i n, k+m}^{*}
$$

where $z_{\text {out }, k}$ is the $k^{\text {th }}$-output sample, $z_{i n, k-m}$ is the $(k-m)^{t h}$-input sample, * denote complex conjugate, $c_{m}$ and $d_{m}$ are compensator coefficients determined based on coupler's frequency dependent magnitude and phase imbalance. The receiver presented in this paper is developed by applying this compensation method.

\section{Proposed receiver diagram}

The proposed wideband receiver diagram is illustrated in Fig. 3. The incoming signal passes through a non-ideal coupler whose magnitude/phase mismatch is designed so that, in each Nyquist zone pair (i.e. Nyquist zone 1-2, zone $3-4$, etc.), it has different magnitude/phase mismatch. The coupler output then passes through several mismatch compensators each of which is designed based on magnitude/phase mismatch spectra in different Nyquist zones. FFT is conducted upon original coupler output and compensator 
outputs. If the correct compensation scheme is applied, then image signals caused by magnitude/phase mismatch can be significantly reduced. On the other hand, the compensator based on inaccurate magnitude/phase mismatch profile will have a compromised performance. Based on the reduction amount of image, the receiver can determine the Nyquist zone of the incoming signal thus its actual frequency. Comparing Fig. 1 and Fig. 3, one can notice that the proposed diagram uses only one ADC and reduces the need for many down-converters. Besides, because we use complex-valued receiver, the aliasing effects around Nyquist frequency can be ignored.

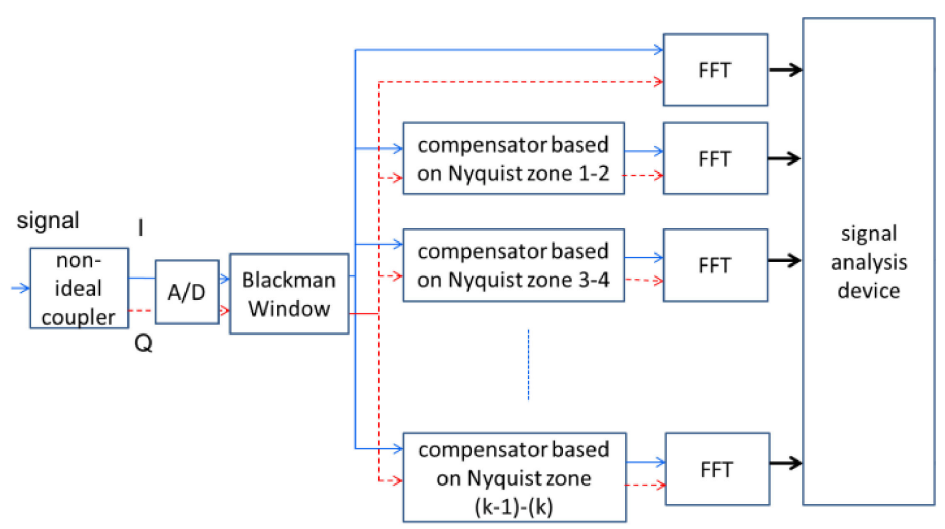

Fig. 3. The proposed signal detection method

\section{Simulation results}

To test the proposed algorithm, we conducted a test in simulation. The coupler magnitude/phase mismatch profile used in the simulation is illustrated in Fig. 4(a) which shows the coupler has different magnitude/phase mismatch profiles is different Nyquist zones. The FFT size is 256 point and sampling frequency is set at $2.56 \mathrm{GHz}$. After the A/D conversion, the Blackman window multiplies both I and Q channel to reduce spectral leakage [1]. The working bandwidth of the receiver is set as $0-15.36 \mathrm{GHz}$ (12 Nyquist zones); therefore, 6 mismatch compensators are designed based on the mismatch profiles of different Nyquist zones. The signal-to-noise ratio is set at $15 \mathrm{~dB}$ and the sinusoidal signal frequency is swept from $10 \mathrm{MHz}$ to $15.36 \mathrm{GHz}$. The signal frequency is determined based on the peak of FFT spectrum and signal's Nyquist zone which is settled by comparing image reduction amount achieved by different compensators. The frequency estimation accuracy can be improved using interpolation between FFT peak and its adjacent FFT bins [3]. The image signal can be identified by its power reduction generated by compensators thus reducing false alarm rate. The resulting frequency estimation error is plotted in Figs. 4(b). As shown in Figs. 4(b) the proposed algorithm can successfully determine signal's frequency over 12 Nyquist zones and frequency estimation error is less than $1.5 \mathrm{MHz}$. Without multiple compensators, the receiver can only cover two Nyquist zones. We focus on signal frequency estimation in this article. Other signal characteritics such as signal 
power, duration, and time-of-arrival can be determined using techniques presented in Ref. [10].

One thing worthy of notice is that, it might be necessary to introduce significant mismatch to make the proposed algorithm feasible. How to implement such a strong frequency dependent magnitude/phase mismatch will be a topic of future study.
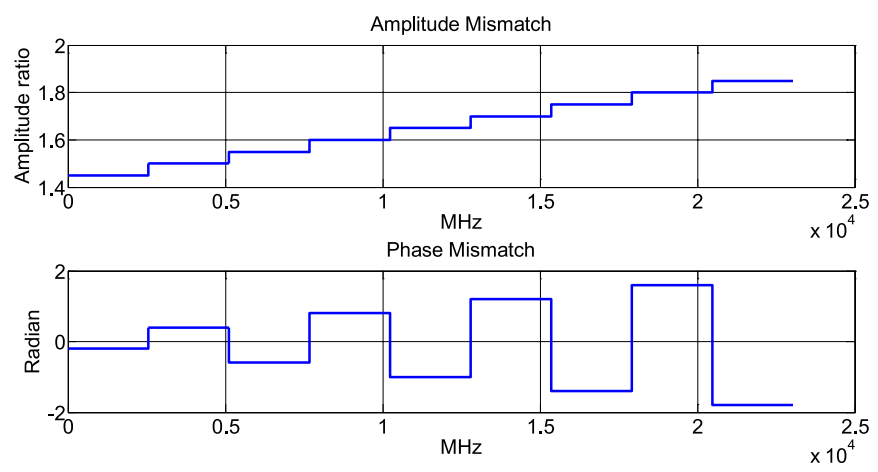

(a)

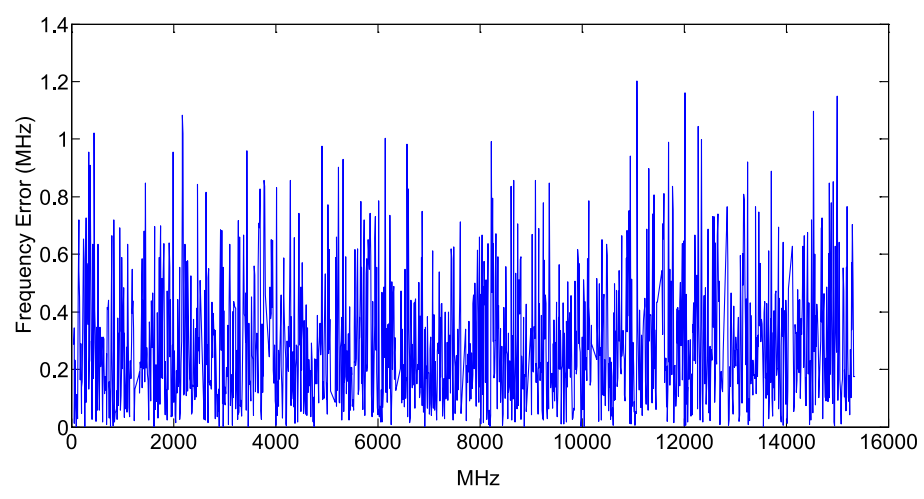

(b)

Fig. 4. (a) The frequency-dependent magnitude/phase mismatch used in simulation (b) frequency estimation error in simulation (Sampling rate: $2.56 \mathrm{GHz}$ )

\section{Conclusion}

A digital wideband signal processing algorithm designed to detect real-valued signal over multiple Nyquist zones is presented. By exploiting frequencydependent magnitude/phase mismatch in the conversion from real-valued signals to complex-valued signals, the proposed algorithm can distinguish signals in different Nyquist zones. The preliminary results presented in this letter demonstrate the validity of the presented method.

\section{Acknowledgments}

The authors would like to thank Dr. Lihyeh Liou, Mr. David Lin, and Dr. James Tsui for valuable opinions and comments. 